\title{
Ab Initio Large-Basis No-Core Shell Model
}

B. R. Barrett, P. Navratil, A. Nogga, W. E. Ormand, I. Stetcu, J. P. Vary, H. Zhan

March 8, 2005

International Conference on Nuclear Data for Science \& Technology Santa Fe, NM, United States September 26, 2004 through October 1, 2004 
This document was prepared as an account of work sponsored by an agency of the United States Government. Neither the United States Government nor the University of California nor any of their employees, makes any warranty, express or implied, or assumes any legal liability or responsibility for the accuracy, completeness, or usefulness of any information, apparatus, product, or process disclosed, or represents that its use would not infringe privately owned rights. Reference herein to any specific commercial product, process, or service by trade name, trademark, manufacturer, or otherwise, does not necessarily constitute or imply its endorsement, recommendation, or favoring by the United States Government or the University of California. The views and opinions of authors expressed herein do not necessarily state or reflect those of the United States Government or the University of California, and shall not be used for advertising or product endorsement purposes. 


\title{
Ab Initio Large-Basis No-Core Shell Model
}

\author{
Bruce R. Barrett ${ }^{*}$, Petr Navrátil ${ }^{\dagger}$, Andreas Nogga $^{* *}$, W. Erich Ormand ${ }^{\dagger}$, \\ Ionel Stetcu*, James P. Vary and Hu Zhan* \\ ${ }^{*}$ Department of Physics, PO Box 210081, University of Arizona, Tucson, AZ 85721 \\ ${ }^{\dagger}$ University of California, Lawrence Livermore National Laboratory, Livermore, CA 94551 \\ ${ }^{* *}$ Institute for Nuclear Theory, University of Washington, Box 351550, Seattle, WA 98195 \\ ${ }^{\ddagger}$ Department of Physics and Astronomy, Iowa State University, Ames, IA 50011
}

\begin{abstract}
We discuss the motivation, theory, and formulation of the ab initio No-Core Shell Model (NCSM). In this method the effective Hamiltonians are derived microscopically from realistic nucleon-nucleon $(N N)$ and theoretical three-nucleon $(N N N)$ potentials, as a function of the finite harmonic-oscillator (HO) basis space. We present converged results for the $A=3$ and 4 nucleon systems, which are in agreement with results obtained by other exact methods, followed by results for $p$-shell nuclei. Binding energies, rms radii, excitation spectra, and electromagnetic properties are discussed. The favorable comparison with available data is a consequence of the underlying $N N$ and $N N N$ interactions rather than a phenomenological fit.
\end{abstract}

\section{INTRODUCTION}

The major outstanding problem in nuclear-structure physics is to calculate the properties of finite nuclei starting from the basic interactions among the nucleons. While various methods have been developed to solve the three- and four-nucleon systems with realistic interactions [1, 2, 3, 4], few approaches are suitable for heavier nuclei at this time. Apart from the coupled cluster method [5] applied typically to closed-shell and nearclosed-shell nuclei, the Green's function Monte Carlo method is the only approach for which exact solutions of systems with $A \leq 10$ have been obtained [4].

For both few-nucleon systems and $p$-shell nuclei, treated as systems of nucleons interacting by realistic NN interactions, we apply the no-core shell-model (NCSM) approach $[6,7,8,9,10,11,12,13,14,15]$

\section{NO-CORE SHELL-MODEL APPROACH}

The NCSM is based on an effective Hamiltonian derived from realistic "bare" interactions and acting within a finite Hilbert space. All $A$-nucleons are treated on an equal footing. In the standard formulation of this approach, utilizing a single-particle (s.p.) coordinate $\mathrm{HO}$ basis, the effective interaction is determined for a system of two (or three) nucleons bound in a $\mathrm{HO}$ well and interacting by the $N N$ potential (and possibly also a $N N N$ potential). We note that the use of a $\mathrm{HO}$ basis is crucial for insuring that the center-of-mass (CM) motion of the nucleus does not mix with the internal motion of the nucleons. This approach is limited by the model-space size as well as by the fact that only a two-body (or threebody) effective interaction is used, despite the fact that higher-body effective interactions might not be negligible. Although the practical applications depend on the $\mathrm{HO}$ frequency and the model-space size, the approach is computationally tractable and our results are guaranteed to converge to an exact solution once a sufficiently large model space is reached $[8,9]$.

In the NCSM approach we start with the one- plus two-body (and possibly three-body) Hamiltonian for the $A$-nucleon system, i.e.,

$$
H_{A}=\sum_{i=1}^{A} \frac{\vec{p}_{i}^{2}}{2 m}+\sum_{j>i=1}^{A} V_{i j}^{N N}
$$

where $m$ is the nucleon mass and $V_{i j}^{N N}$ is the $N N$ interaction. In the next step we modify the Hamiltonian (1) by adding to it the CM HO potential $\frac{1}{2} A m \Omega^{2} \vec{R}^{2}$, $\vec{R}=\frac{1}{A} \sum_{i=1}^{A} \vec{r}_{i}$. This potential will be subtracted in the final many-body calculation. The modified Hamiltonian, with a pseudo-dependence on the $\mathrm{HO}$ frequency $\Omega$, can be cast into the form

$$
\begin{aligned}
H_{A}^{\Omega} & =\sum_{i=1}^{A}\left[\frac{\vec{p}_{i}^{2}}{2 m}+\frac{1}{2} m \Omega^{2} \vec{r}_{i}^{2}\right] \\
& +\sum_{j>i=1}^{A}\left[V_{i j}^{N N}-\frac{m \Omega^{2}}{2 A}\left(\vec{r}_{i}-\vec{r}_{j}\right)^{2}\right] .
\end{aligned}
$$


This added and later subtracted potential permits the use of the convenient $\mathrm{HO}$ basis and provides a mean field that facilitates the calculation of the effective interactions.

Since we solve the many-body problem in a finite HO model space, the realistic nuclear interaction in Eq. (2) will yield unreasonable results unless we employ a model-space-dependent effective Hamiltonian. In general, for an $A$-nucleon system, an $A$-body effective interaction is needed. As we will discuss later, the effective interaction is, in the present calculations, approximated by a two-body or a three-body effective interaction. Large model spaces are desirable to minimize the role of neglected effective many-body terms. In fact, large model spaces are desirable for the evaluation of any observable, i.e., the larger the model space, the smaller the renormalization contributions to any effective operator.

As the Hamiltonian $H_{A}^{\Omega}(2)$ differs from the Hamiltonian $H_{A}$ (1) only by a CM term, no dependence on $\Omega$ should exist for the intrinsic properties of the nucleus. However, because of the neglect of many-body terms in the effective-interaction derivation, a dependence on $\Omega$ appears in our calculations. This dependence decreases as the size of the model-space is increased.

For the derivation of the effective interaction, we adopt approaches presented by Lee and Suzuki [16], Da Providencia and Shakin [17], and Suzuki and Okamoto [18], which yield an Hermitian effective Hamiltonian.

In the spirit of the above-mentioned papers, we introduce a unitary transformation of the Hamiltonian, by choosing an anti-Hermitian operator $S$, such that

$$
\mathscr{H}=e^{-S} H_{A}^{\Omega} e^{S} .
$$

In our approach, $S$ is determined by the requirements that $\mathscr{H}$ and $H_{A}^{\Omega}$ have the same symmetries and eigenspectra over the subspace $\mathscr{K}$ of the full Hilbert space. In general, both $S$ and the transformed Hamiltonian are $A$-body operators. Our simplest, non-trivial approximation to $\mathscr{H}$ is to develop a two-body effective Hamiltonian. This approach consists then of an approximation to a particular level of clustering:

$$
\mathscr{H} \approx \mathscr{H}^{(1)}+\mathscr{H}^{(a)}
$$

where the one-body and $a$-body $(a \leq A)$ pieces are given as

$$
\begin{aligned}
\mathscr{H}^{(1)} & =\sum_{i=1}^{A} h_{i}, \\
\mathscr{H}^{(a)} & =\frac{\left(\begin{array}{l}
A \\
2
\end{array}\right)}{\left(\begin{array}{l}
A \\
a
\end{array}\right)\left(\begin{array}{l}
a \\
2
\end{array}\right)} \sum_{i_{1}<i_{2}<\ldots<i_{a}}^{A} \tilde{V}_{i_{1} i_{2} \ldots i_{a}},
\end{aligned}
$$

with

$$
\tilde{V}_{12 \ldots a}=e^{-S^{(a)}} H_{a}^{\Omega} e^{S^{(a)}}-\sum_{i=1}^{a} h_{i}
$$

where $S^{(a)}$ is an $a$-body operator;

$$
H_{a}^{\Omega}=h_{1}+h_{2}+h_{3}+\ldots+h_{a}+V_{a} ;
$$

and

$$
V_{a}=\sum_{i<j}^{a} V_{i j}
$$

Note that there is no sum over $a$ in Eq. (4), because $\mathscr{H}^{(a)}$ is not a series expansion. In the above equations, it has been assumed that the basis states are eigenstates of the one-body, in our case HO, Hamiltonian $\sum_{i=1}^{A} h_{i}$. We now introduce our present application, in which we take $a=2$, so that the interaction, $\tilde{V}_{12}$, is given by Eq. (7):

$$
\tilde{V}_{12}=e^{-S^{(2)}}\left(h_{1}+h_{2}+V_{12}\right) e^{S^{(2)}}-\left(h_{1}+h_{2}\right) .
$$

The full space is divided into a model or P-space, and a Q-space, using the projectors $P$ and $Q$ with $P+Q=1$. It is then possible to determine the transformation operator $S^{(2)}$ from the decoupling condition

$$
Q_{2} e^{-S^{(2)}}\left(h_{1}+h_{2}+V_{12}\right) e^{S^{(2)}} P_{2}=0 .
$$

The two-nucleon-state projectors $\left(P_{2}, Q_{2}\right)$ follow from the definitions of the $A$-nucleon projectors $P, Q$. The solution for this approach [18] is given by

$$
S^{(2)}=\operatorname{arctanh}\left(\omega-\omega^{\dagger}\right),
$$

with the operator $\omega$ satisfying $\omega=Q_{2} \omega P_{2}$. This is the same operator, which we previously employed $[7,8,9]$. It can be directly obtained from the eigensolutions $|k\rangle$ of $h_{1}+h_{2}+V_{12}$ as $\left\langle\alpha_{Q}|\omega| \alpha_{P}\right\rangle=\sum_{k \in \mathscr{K}}\left\langle\alpha_{Q} \mid k\right\rangle\left\langle\tilde{k} \mid \alpha_{P}\right\rangle$, where we denote by tilde the inverted matrix of $\left\langle\alpha_{P} \mid k\right\rangle$. In the above relation, $\left|\alpha_{P}\right\rangle$ and $\left|\alpha_{Q}\right\rangle$ are the two-particle model-space and Q-space basis states, respectively, and $\mathscr{K}$ denotes a set of $d_{P}$ eigenstates, whose properties are reproduced in the model space, with $d_{P}$ equal to the model-space dimension.

With the help of the solution for $\omega$ we obtain a simple expression for the matrix elements of the Hermitian effective Hamiltonian

$$
\begin{aligned}
\left\langle\alpha_{P}\left|\bar{H}_{\mathrm{a}-\mathrm{eff}}\right| \alpha_{P}^{\prime}\right\rangle= & \sum_{k \in \mathscr{K}} \sum_{\alpha_{P}^{\prime \prime}} \sum_{\alpha_{P}^{\prime \prime \prime}}\left\langle\alpha_{P}\left|\left(P_{a}+\omega^{\dagger} \omega\right)^{-1 / 2}\right| \alpha_{P}^{\prime \prime}\right\rangle \\
& \times\left\langle\alpha_{P}^{\prime \prime} \mid \tilde{k}\right\rangle E_{k}\left\langle\tilde{k} \mid \alpha_{P}^{\prime \prime \prime}\right\rangle \\
& \times\left\langle\alpha_{P}^{\prime \prime \prime}\left|\left(P_{a}+\omega^{\dagger} \omega\right)^{-1 / 2}\right| \alpha_{P}^{\prime}\right\rangle . \quad(13)
\end{aligned}
$$

The two-body effective interaction $P_{2} \tilde{V}_{12} P_{2}$ can be obtained from Eq. (13) by subtracting the one-body piece and depends on $A$, on the HO frequency $\Omega$ and on $N_{\max }$, the maximum many-body $\mathrm{HO}$ excitation energy (above the lowest configuration) defining the P-space. It follows that $\mathscr{H}^{(1)}+\mathscr{H}^{(2)}-H_{\mathrm{CM}}$ is translationally invariant and that $\tilde{V}_{12} \rightarrow V_{12}$ for $N_{\max } \rightarrow \infty$. 
The most significant approximation used in the present application is the neglect of higher than two-body clusters in the unitary transformed Hamiltonian expansion. Because our method is not a variational approach, the neglected clusters can contribute either positively or negatively to the binding energy. Our approach can be readily generalized in order to include, e.g., three-body clusters, and to demand the model-space decoupling on the threebody cluster level. A method for deriving the three-body effective interaction was presented in our papers $[8,9]$, which can be obtained by setting $a=3$ in Eqs. (4)-(9).

In the limit $a \rightarrow A$, we obtain the exact solutions for $d_{P}$ states of the full problem for any finite basis space. On account of our cluster approximation, a dependence of our results on the model-space size $N_{\max }$ and on the HO frequency $\Omega$ arises. The residual $N_{\max }$ and $\Omega$ dependences can be used to infer the uncertainty in our results.

In order to construct the operator $\omega$ we need to select the set of eigenvectors $\mathscr{K}$. Because of the added CM Hamiltonian, the $a$-body clusters are confined, which ensures that all eigenvectors are bound states. We keep the lowest states obtained in each two-body channel. It turns out that these states also have the largest overlap with the model space for the range of $\hbar \Omega$ and the Pspaces we have investigated.

We input the effective Hamiltonian, now consisting of a relative 2-body operator and the pure $H_{C M}$ term introduced earlier, into an $\mathrm{m}$-scheme Lanczos diagonalization process to obtain the P-space eigenvalues and eigenvectors, e.g., the Many-Fermion Dynamics (MFD) shellmodel code [19]. At this stage we also add the term $H_{C M}$ again with a large positive coefficient to separate the physically interesting states with $0 s \mathrm{CM}$ motion from those with excited CM motion. We retain only the states with pure $0 s \mathrm{CM}$ motion when evaluating observables. All observables that are expressible as functions of relative coordinates, such as the rms radius and radial densities, are then evaluated free of CM motion effects.

We also have a second diagonalization code in Jacobi coordinates and a $\mathrm{HO}$ basis $[8,9]$, for which three- and even higher-body interactions can be more easily employed. It should be stressed, however, that these two alternative approaches are completely equivalent and lead to the same results.

We close our presentation on the theoretical framework by noting that all observables require the same transformation as implemented on the Hamiltonian. We have found rather small effects on the rms radius operator when we transformed it to a P-space effective rms operator at the $a=2$ cluster level [10]. On the other hand, substantial renormalization was obtained for the kinetic energy operator when using the $a=2$ transformation to evaluate its expectation value [20]. Recent results [21] indicate that at the $a=2$ cluster level the amount of renormalization is sensitive to the range of the operator.
TABLE 1. Results for the ground-state (g.s.) energies (in MeV) obtained for ${ }^{3} \mathrm{H},{ }^{3} \mathrm{He}$ and ${ }^{4} \mathrm{He}$ using the CD-Bonn, AV18 and AV8' NN potentials. The AV8' results are without the Coulomb interaction. Shown values are based on the results calculated in the model spaces up to $N_{\max }=50$ for ${ }^{3} \mathrm{H},{ }^{3} \mathrm{He}$, and $N_{\text {max }}=18$ for ${ }^{4} \mathrm{He}$, respectively. The errors were estimated from the dependences on the HO frequency $\Omega$ and on the model-space size $N_{\max }$.

\begin{tabular}{lrrr}
\hline & CD-Bonn & NN potential & \\
\hline${ }^{3} \mathrm{H}$ & $-8.002(4)$ & $-7.61(1)$ & $-7.75(2)$ \\
\hline${ }^{3} \mathrm{He}$ & $-7.249(4)$ & $-6.90(1)$ & $-7.75(2)$ \\
\hline${ }^{4} \mathrm{He}$ & $-26.30(15)$ & & $-25.80(20)$ \\
\hline
\end{tabular}

\section{RESULTS}

We performed calculations for the $A=3$ systems interacting by several realistic $N N$ potentials in model spaces up to $50 \hbar \Omega\left(N_{\max }=50\right)$. We employed the modern, realistic CD-Bonn [22], AV18 and AV8' [4] NN potentials. Our ${ }^{3} \mathrm{H}$ and ${ }^{3} \mathrm{He}$ results are summarized in Table 1 . The non-local CD-Bonn NN potential has a weaker tensor force than the local AV18 and AV8'. In general, we observe that the stronger the tensor force is, the stronger the HO frequency dependence and the slower the convergence with $N_{\max }$. In particular, even for the AV18 NN potential, the $N_{\max }=50$ models space is sufficient for obtaining a converged result with an error less than $10 \mathrm{keV}$, as shown in Fig. 1(a). It is seen that the utilization of the effective $N N$ interaction speeds up the convergence significantly compared with the bare interaction. The AV8' $N N$ potential is more difficult and some HO frequency dependence remains even at $50 \hbar \Omega$. Our overall $A=3$ results, however, are in excellent agreement with other exact methods, as can be judged by comparing numbers from Table 1 with results presented in $[23,24]$ and references therein.

Also shown in Table 1 are our ${ }^{4} \mathrm{He}$ results obtained in model spaces up to $18 \hbar \Omega$. This model-space size allowed us to bring our CD-Bonn ${ }^{4} \mathrm{He}$ results into even better agreement with the Faddeev-Yakubovsky calculations by Nogga et al. [24]. For the CD-Bonn potential our results are essentially converged, while for the AV8' or AV18 potentials, convergence is slower. For AV8' we used the three-body effective interaction, which improved the convergence $[8,9]$.

A bigger challenge for the NCSM is the $p$-shell, where model spaces increase rapidly in size with increasing $N_{\max }$. Consequently, model spaces larger than $N_{\max }=8$ are not presently feasible for most $p$-shell nuclei [14] However, besides increasing $N_{\max }$ to improve convergence, one can also increase the cluster size of the effec- 

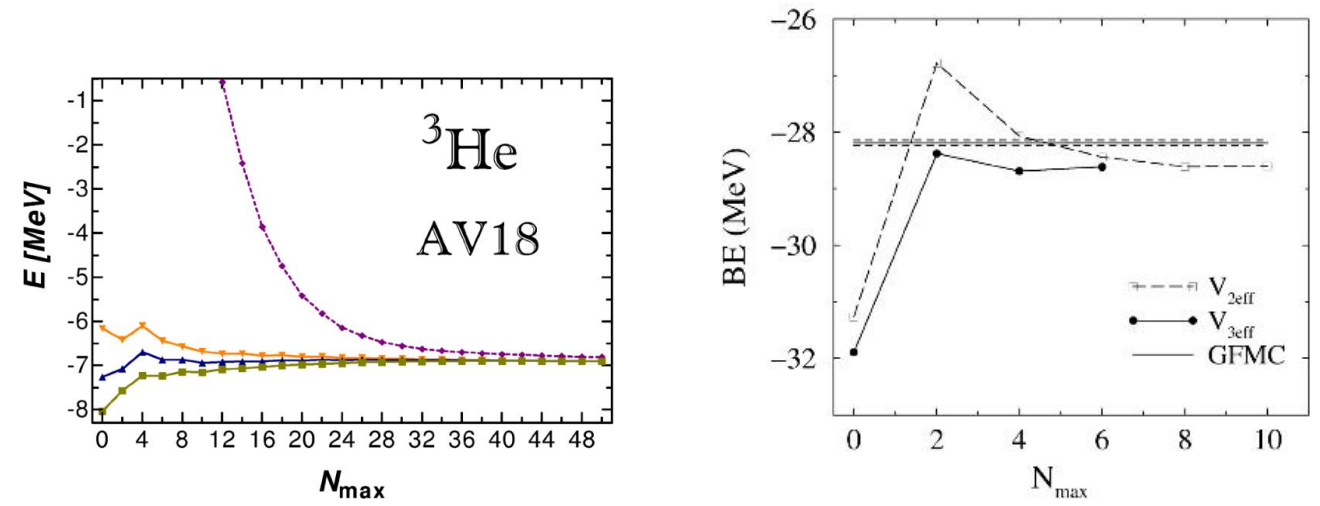

(a)

(b)

FIGURE 1. (a) Ground-state energy dependence on the model-space size for ${ }^{3} \mathrm{He}$ interacting by the AV18 NN potential. The dashed line shows the result based on the bare interaction. The solid lines with down-pointing triangles, up-pointing triangles and squares are results based on the effective interaction for $\hbar \Omega=32,28$ and $24 \mathrm{MeV}$, respectively. (b) Ground-state energy dependence on the model-space size for ${ }^{6} \mathrm{Li}$ using the AV8' $N N$ potential with Coulomb. The plotted energies occur at the HO frequency minima for the given value of $N_{\max }$ The results using both the two-body and the three-body effective interaction are compared with the GFMC results from [4]. The figure is from [12].

tive interaction. This has been investigated by Navrátil and Ormand [12] for several $p$-shell nuclei. E.g., for ${ }^{6} \mathrm{Li}$, it was demonstrated that three-body effective interactions accelerate convergence. This is shown in Fig. 1(b)

Our ability to calculate the effective Hamiltonian at the three-body cluster level as well as for the two-body cluster makes it possible for us to investigate the nature of different $N N N$ interaction models [25]. The spectra of the light nuclei are well suited for analyzing $N N N$ forces, because they are especially sensitive to their spin/isospin structure. In addition, the NCSM results for the spectra typically converge faster than the binding energies (see, e.g., [10]) and are generally more accurately predicted than the binding energies. To exemplify the sensitivity of the spectra to the $N N N$ interaction, we show in Fig. 2(a) our results for the excitation energy of the $3^{+}$state in ${ }^{6} \mathrm{Li}$. $N N$ forces alone overpredict this observable. Note that the combinations AV8' with the $2 \pi$ exchange TucsonMelbourne (TM'(99)) $N N N$ force [26] are already close to the experimental number. This becomes even more pronounced for ${ }^{10} \mathrm{~B}$, where TM'(99) corrects the wrong ordering of ground and excited state predicted by $N N$ interactions only or in combination with the Urbana-IX $N N N$ force [4]. We also studied the chiral $N N$ interaction Idaho-N3LO [27] in combination with the consistently defined leading chiral $N N N$ interaction [28]. We identified two sets of parameters, which describe the ${ }^{3} \mathrm{H}$ and ${ }^{4} \mathrm{He}$ binding energies equally well. The excitation energy is different for both sets of parameters, showing the sensitivity of this quantity to the $N N N$ force structure. Calculations for other nuclei are in progress.

We next address a vastly more complex system, ${ }^{12} \mathrm{C}$. There are several pressing reasons to investigate ${ }^{12} \mathrm{C}$ in a
TABLE 2. Experimental and calculated g.s. and $3^{-} 0$-state energies, point-proton rms radii, the $2_{1}^{+}$-state and the $3^{-0}$ state quadrupole moments of ${ }^{12} \mathrm{C}$. Results obtained in different model spaces, i.e., $N_{\max }=6,4,2$ for the positive-parity and $N_{\max }=5,3,1$ for the negative-parity states and using effective interactions derived from the CD-Bonn $\mathrm{NN}$ potential are given. The calculated excitation energy of the $3^{-} 0$ state is obtained by comparing its energy in the $N \hbar \Omega$ space with the ground state in the $(N-1) \hbar \Omega$ space. A HO frequency of $\hbar \Omega=15 \mathrm{MeV}$ was employed. The experimental values are from $[32,33]$.

\begin{tabular}{lrrrr}
\hline & ${ }^{12} \mathrm{C}$ & CD-Bonn \\
\hline model space & - & $6 \hbar \Omega$ & $4 \hbar \Omega$ & $2 \hbar \Omega$ \\
\hline$\left|E_{\mathrm{gs}}\left(0^{+} 0\right)\right|[\mathrm{MeV}]$ & 92.162 & 85.630 & 88.518 & 92.353 \\
$r_{p}[\mathrm{fm}]$ & $2.35(2)$ & 2.195 & 2.199 & 2.228 \\
$Q_{2^{+}}\left[e \mathrm{fm}^{2}\right]$ & $+6(3)$ & 4.717 & 4.533 & 4.430 \\
\hline model space & - & $5 \hbar \Omega$ & $3 \hbar \Omega$ & $1 \hbar \Omega$ \\
\hline$\left|E\left(3^{-} 0\right)\right|[\mathrm{MeV}]$ & 82.521 & 72.952 & 75.331 & 83.390 \\
$r_{p}[\mathrm{fm}]$ & & 2.309 & 2.316 & 2.425 \\
$Q_{3^{-}}\left[e \mathrm{fm}^{2}\right]$ & & -7.942 & -7.596 & -6.936 \\
$E\left(3^{-} 0\right)-E_{\mathrm{gs}}[\mathrm{MeV}]$ & 9.641 & 15.566 & 17.022 & 21.557 \\
\hline
\end{tabular}

way that preserves as much predictive power as possible. The ${ }^{12} \mathrm{C}$ nucleus plays an important role [29] in neutrino studies using liquid scintillator detectors. Also, there has been considerable interest recently in parity-violating electron scattering from $\left(J^{\pi}, T\right)=\left(0^{+}, 0\right)$ targets, like ${ }^{12} \mathrm{C}$, to measure the strangeness content of the nucleon $[30,31]$.

To solve for the properties of ${ }^{12} \mathrm{C}$, we employ the $\mathrm{m}$ scheme MFD code [19]. Here we give an extension of our ${ }^{12} \mathrm{C}$ study published in [10] to a $6 \hbar \Omega$ space, where the dimensions reach 32 million. We utilize $\hbar \Omega=15 \mathrm{MeV}$, which lies in the range where the largest model-space 

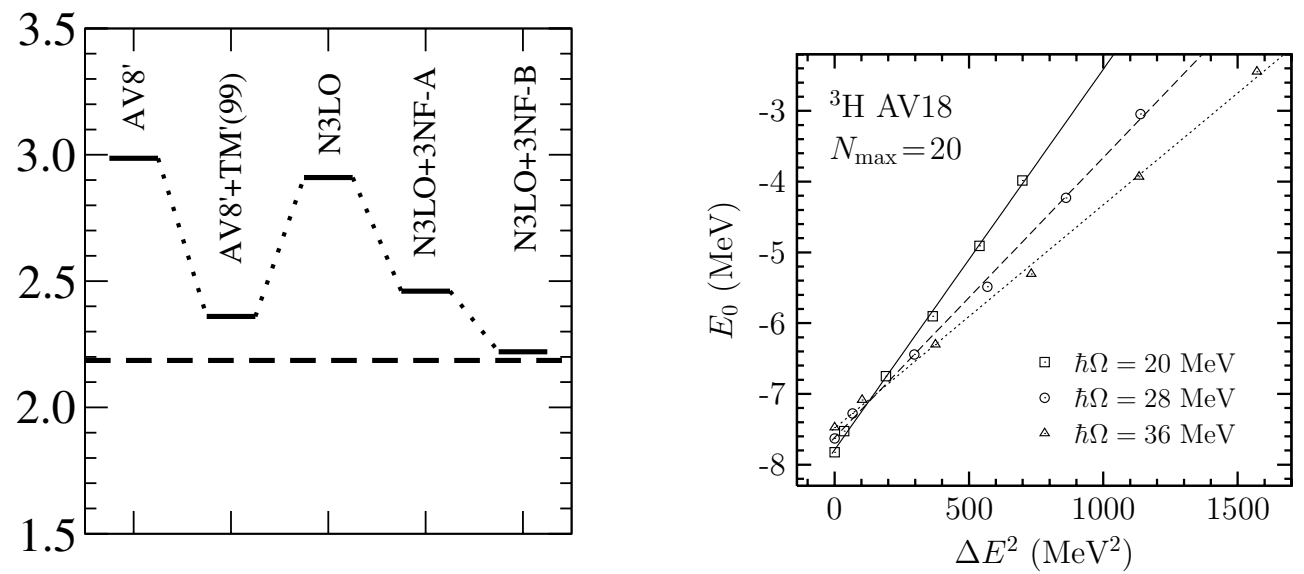

(a)

(b)

FIGURE 2. (a) Excitation energy of the $3^{+}$state of ${ }^{6} \mathrm{Li}$ for $N N$ and $N N N$ interactions. The dashed line marks experiment. All results are for $N_{\max }=6$. (b) The linear relation between $E_{0}$ and $\Delta E^{2}$, for ${ }^{3} \mathrm{H}$ with the $A V 18 N N$ potential. Symbols from right to left correspond to $\tilde{N}_{\max }=10,12, \ldots, 20$ for each model. The lines fit the results from $\tilde{N}_{\text {max }}=10$ to 18 .

results are least sensitive to $\hbar \Omega[10]$.

In Table 2 we present the g.s. and $3^{-} 0$ energies as well as several other observable results calculated with the CD-Bonn $N N$ potential. While the energy of the g.s. eigenstate increases with increasing model space, we find that the relative level spacings are less dependent on model-space size. In particular, the excitation spectrum is remarkably stable when the model space is changed from $4 \hbar \Omega$ to $6 \hbar \Omega$. In general, we obtain reasonable agreement of the states dominated by $0 \hbar \Omega$ configurations with experimental levels. Our obtained binding energy of about $85.6 \mathrm{MeV}$ in the $6 \hbar \Omega$ space is expected to decrease with a further model-space enlargement. We estimate, however, that our result should be within better than $10 \%$ of the exact solution for the two-body CD-Bonn $N N$ potential. In order to reach the experimental binding energy, a $N N N$ interaction is likely necessary [4].

The two- or higher- $\hbar \Omega$ dominated states, such as the $7.65 \mathrm{MeV} 0^{+} 0$ state, are not seen in the low-lying part of our calculated spectra. Overall, the convergence rate of the $2 \hbar \Omega$ dominated states is quite different than that of the ground state. We find a decreasing excitation energy of the second $0^{+} 0$ state. We expect this state eventually to change its structure and become the cluster state.

To conclude this section, we note that it is desirable to further increase the model-space sizes of these calculations, so as to improve the accuracy of our predictions and to enable us to investigate the properties of heavier mass nuclei. We recently developed an extrapolation method for estimating the binding energies of NCSM calculations without diagonalizing the complete Hamiltonian in extremely large basis spaces [34]. It is motivated by the observation that the binding energy $E_{0}=\langle H\rangle$ evaluated in an approximate ground state must approach the exact binding energy as the energy variance $\Delta E^{2}=\left\langle H^{2}\right\rangle-\langle H\rangle^{2}$ vanishes [35]. From second-order perturbation theory, we expect that there exists an approximate linear correlation between $E_{0}$ and $\Delta E^{2}$, if approximate ground states are calculated from a Hamiltonian truncated from the large-space Hamiltonian by $\mathrm{HO}$ quantum numbers $\tilde{N}_{\text {max }}$. This linear scaling is used to extrapolate large-space results from smaller-space calculations in the context of the NCSM. We obtained the result that the converged binding energy scales with the energy variance $\Delta E^{2}$, as shown in Fig. 2(b) for ${ }^{3} \mathrm{H}$. Compared to direct diagonalization, the extrapolation has an error of a few tens of $\mathrm{keV}$ for ${ }^{3} \mathrm{H}$. A large value of $N_{\max }$ will further reduce the extrapolation error. We are presently extending this procedure to heavier mass nuclei.

\section{CONCLUSIONS}

In this contribution, we described the ab initio NCSM approach and discussed its application to the lightest nuclei, ${ }^{3} \mathrm{H},{ }^{3} \mathrm{He}$ and ${ }^{4} \mathrm{He}$, for which we obtain wellconverged results. Due to the utility of Jacobi coordinates in these few-nucleon calculations, we are able to reach very large model spaces, i.e., $50 \hbar \Omega$ for $A=3$ and $18 \hbar \Omega$ for $A=4$. We also showed results for ${ }^{6} \mathrm{Li}$ and ${ }^{12} \mathrm{C}$. For the accurate description of $p$-shell nuclei not only $N N$, but also $N N N$ interactions are important. In order to include the latter, we need three-body effective interactions. These also improve the rate of convergence in smaller model spaces, although more work remains to be done regarding the role and properties of $N N N$ and perhaps $N N N N$ effective interactions. We have shown how the NCSM approach at the $N N N$ cluster level can be 
used to analyze the nature of different theoretical models for the $N N N$ forces. Investigations of the renormalization properties of other physical operators besides the Hamiltonian indicate that at the two-body cluster level the amount of renormalization is sensitive to the range of the operator. We have established an extrapolation method to extend our calculations to even larger model spaces. It can serve as a way to estimate the uncertainties of NCSM results arising from $\hbar \Omega$ dependence and choices of interactions. It should be noted that our calculations contain no adjustable parameters. The favorable comparison with available data that we obtain is a consequence of the underlying $N N$ and $N N N$ interactions.

\section{ACKNOWLEDGMENTS}

B.R.B., I.S., and H.Z. acknowledge partial support by NSF grants PHY0070858 and PHY0244389. J.P.V. acknowledges partial support by USDOE grant No. DEFG-02-87ER-40371. The work was performed in part under the auspices of the U.S. Department of Energy by the University of California, Lawrence Livermore National Laboratory under contract No. W-7405-Eng-48. P.N. and W.E.O. received support from LDRD contract 00-ERD-058. A.N. acknowledges support by USDOE grants DE-F02-01ER41187 and DE-FG02-00ER41132. B.R.B. thanks B.K. Jennings for helpful discussions and TRIUMF for its hospitality during the preparation of this contribution.

\section{REFERENCES}

1. L. D. Faddeev, Zh. Eksp. Teor. Fiz. 39, 1459 (1960) Sov. Phys.-JETP 12, 1014 (1961); J.L. Friar, G.L. Payne, V.G.J. Stoks, and J.J. de Swart, Phys. Lett. B 311, 4 (1993).

2. O. A. Yakubovsky, Sov. J. Nucl. Phys. 5, 937 (1967); W. Glöckle and H. Kamada, Phys. Rev. Lett. 71, 971 (1993).

3. M. Viviani, A. Kievsky, and S. Rosati, Few-Body Systems 18, 25 (1995).

4. R. B. Wiringa, V. G. J. Stoks and R. Schiavilla, Phys. Rev. C 51, 38 (1995); B. S. Pudliner, V. R. Pandharipande, J. Carlson, S. C. Pieper and R. B. Wiringa, Phys. Rev. C 56 1720, (1997); R. B. Wiringa, Nucl. Phys. A 631, 70c (1998); S. Pieper and R. B. Wiringa, Annu. Rev. Nucl. Part. Sci. 51, 53 (2001); S. Pieper, K. Varga and R. B. Wiringa, Phys. Rev. C 66, 044310 (2002).

5. R. F. Bishop, M. F. Flynn, M. C. Boscá, E. Buendía and R. Guardiola, Phys. Rev. C 42, 1341 (1990); J. H. Heisenberg and B. Mihaila, ibid. 59, 1440 (1999).

6. D. C. Zheng, B. R. Barrett, L. Jaqua, J. P. Vary, and R. L. McCarthy, Phys. Rev. C 48, 1083 (1993); D. C. Zheng, J. P. Vary, and B. R. Barrett, Phys. Rev. C 50, 2841 (1994); D. C. Zheng, B. R. Barrett, J. P. Vary, W. C. Haxton, and C. L. Song, Phys. Rev. C 52, 2488 (1995).
7. P. Navrátil and B. R. Barrett, Phys. Rev. C 54, 2986 (1996); Phys. Rev. C 57, 3119 (1998).

8. P. Navrátil and B. R. Barrett, Phys. Rev. C 57, 562 (1998), Phys. Rev. C 59, 1906 (1999).

9. P. Navrátil, G. P. Kamuntavičius and B. R. Barrett, Phys. Rev. C 61, 044001 (2000). E-print archive No. nucl-th/9907054.

10. P. Navrátil, J. P. Vary and B. R. Barrett, Phys. Rev. Lett. 84, 5728 (2000); Phys. Rev. 62, 054311 (2000).

11. P. Navrátil, J. P. Vary and B. R. Barrett, to be published in Phys. Rev. Lett. 87, (22 October 2001).

12. P. Navrátil and W. E. Ormand, Phys. Rev. Lett. 88, 152502 (2002).

13. C. P. Viazminsky and J. P. Vary, J. Math. Phys., 92, 2055 (2001).

14. P. Navrátil and W. E. Ormand, Phys. Rev. C 68, 034305 (2003).

15. P. Navrátil and E. Caurier, Phys. Rev. C 69, 014311 (2004).

16. K. Suzuki and S.Y. Lee, Prog. Theor. Phys. 64, 2091 (1980); K. Suzuki, Prog. Theor. Phys. 68, 246 (1982). K. Suzuki and R. Okamoto, Prog. Theor. Phys. 70, 439 (1983).

17. J. Da Providencia and C. M. Shakin, Ann. of Phys. 30, 95 (1964).

18. K. Suzuki, Prog. Theor. Phys. 68, 1999 (1982); K. Suzuki and R. Okamoto, Prog. Theor. Phys. 92, 1045 (1994).

19. J. P. Vary, "The Many-Fermion-Dynamics Shell-Model Code," Iowa State University (1992) (unpublished); J. P. Vary and D.-C. Zheng, ibid., (1994).

20. H. Kamada, et. al, Phys. Rev. C 64, 044001. (2001).

21. I. Stetcu, B.R. Barrett, P. Navrátil and C. W. Johnson, Conf. Proc. "Blueprint for the Nucleus," Istanbul, Turkey, May 17-22, 2004, to be published in Int. J. Mod. Phys. E (2004).

22. R. Machleidt, F. Sammarruca and Y. Song, Phys. Rev. C 53, 1483 (1996).

23. N. Barnea, W. Leidemann and G. Orlandini, Phys. Rev. C 61, 054001 (2000).

24. A. Nogga, H. Kamada and W. Glöckle, Phys. Rev. Lett. 85, 944 (2000), E-print archive nucl-th/0004023.

25. A. Nogga, E. Epelbaum, P. Navrátil, W. Glöckle, H. Kamada, Ulf-G. Meissner, H. Witała, B.R. Barrett, and J.P. Vary, Nucl. Phys. A737, 236 (2004).

26. S. A. Coon and H. K. Han, Few Body Systems 30, 131 (2001).

27. D. R. Entem and R. Machleidt, Phys. Rev. C 68, 041001(R) (2003).

28. U. van Kolck, Phys. Rev. C 49, 2932 (1994); E. Epelbaum et al., Phys. Rev. C 66, 064001 (2002).

29. A. C. Hayes, Phys. Rep. 315, 257 (1999); P. Vogel, E-print archive nucl-th/9901027; E. Kolbe, K. Langanke and P. Vogel, Nucl. Phys. A 652, 91 (1999).

30. M. J. Musolf, et al., Phys. Rep. 239, 1 (1994).

31. W. E. Ormand, Phys. Rev. Lett. 82, 1101 (1999).

32. F. Ajzenberg-Selove, Nucl. Phys. A506, 1 (1990).

33. I. Tanihata, T. Kobayashi, O. Yamakawa, S. Shimoura, K. Ekuni, K. Sugimoto, N. Takahashi, T. Shimoda and H. Sato, Phys. Lett. B 206, 592 (1988).

34. H. Zhan, A. Nogga, B. R. Barrett, J. P. Vary and P. Navrátil, Phys. Rev. C 69, 034302 (2004).

35. T. Mizusaki and M. Imada, Phys. Rev. C 65, 064319 (2002); Phys. Rev. C 67, 041301(R) (2004). 